\title{
FINITE GROUPS WITH A FAITHFUL REAL-VALUED \\ IRREDUCIBLE CHARACTER WHOSE SQUARE HAS EXACTLY TWO DISTINCT IRREDUCIBLE CONSTITUENTS
}

\author{
EMmanuel ZhMUd
}

Kharkiv State University, Ukraine title.

ABstract. We study the groups satisfying the property stated in the

\section{MAIN RESULT}

To state our results we recall some notation and definitions. In what follows, $G$ is a finite group.

Let $\operatorname{Irr}(G)$ be the set of irreducible complex characters of a group $G$. If $\chi, \psi$ are characters of $G$, we define $\chi \psi$ as function sending $g \in G$ in $\chi(g) \psi(g)$, in particular, $(\chi \chi)(g)=\chi^{2}(g)=\chi(g)^{2}$. It is known that $\chi \psi$ is a character of $G$ if $\chi, \psi$ are. Next, if $\chi$ is a character of $G$, then $\chi^{(2)}(g)=\chi\left(g^{2}\right)$. It is known that the function $\chi^{(2)}$ is a generalized character of $G$ (a generalized character of $G$ is said to be a difference of two characters of $G$, i.e., a linear combination of irreducible characters of $G$ with integer coefficients); see [BZ, $\S 4.6$. We have $\chi^{(2)}(1)=\chi\left(1^{2}\right)=\chi(1)$. If $\theta$ is a generalized character of $G$, then $\operatorname{Irr}(\theta)=\{\chi \in \operatorname{Irr}(\mathrm{G}) \mid\langle\theta, \chi\rangle \neq 0\}$. It is known that if $\chi \in \operatorname{Irr}(\mathrm{G})$, then $\operatorname{Irr}\left(\chi^{(2)}\right) \subseteq \operatorname{Irr}\left(\chi^{2}\right)$ (see $\left.[\mathrm{BZ}, \S 4.6]\right)$. By $1_{G}$ we denote the principal character of $G$.

We use the notation standard for this part of finite group theory (see [1]).

Let, in this paragraph, $\tau$ be a character of $G$ and $\chi \in \operatorname{Irr}(\mathrm{G})$. By $\mathrm{T}_{\tau}=$ $\{g \in G \mid \tau(g)=0\}$ we denote the set of zeros of $\tau$. By Burnside, if $\chi$

2010 Mathematics Subject Classification. 20 D15.

Key words and phrases. Faithful irreducible character, square of irreducible characters, zeros of characters.

Y. Berkovich prepared this paper using the letter of Professor Zhmud (1918-2007) written about 10 years ago. 
is nonlinear, then the set $\mathrm{T}_{\chi}$ is not empty. Set $R(\chi)=G-\mathrm{T}_{\chi}=\{g \in$ $G \mid \chi(g) \neq 0\}$, i.e., $R(\chi)$ is the set of elements of $G$ on which $\chi$ does not vanish. If $D$ is a representation of $G$ affording the character $\tau$, then the function $\operatorname{det}(\tau)$ is defined as follows: $\operatorname{det}(\tau)(g)=\operatorname{det}(D(g))(g \in G)$. We write $\mathrm{Z}(\chi)=\{g \in G|| \chi(g) \mid=\chi(1)\}$; then $\mathrm{Z}(\chi)$ is a normal subgroup of $G$ containing $\operatorname{ker}(\chi)$ and such that $\mathrm{Z}(\chi) / \operatorname{ker}(\chi)=\mathrm{Z}(\mathrm{G} / \operatorname{ker}(\chi))$. By $\operatorname{SL}(2, \mathbf{C})$ $(\mathrm{GL}(2, \mathbf{C}))$ we denote the 2-dimensional special linear (general linear) group over the field $\mathbf{C}$ of complex numbers.

There are few papers in which the nonabelian groups containing a faithful irreducible character $\chi$ such that its square has a special decomposition in one or two irreducible constituents are treated (see [2] and [4]). For example, Isaacs and Zisser [4] have considered the groups $G$ containing a faithful irreducible character $\chi$ such that (i) $\chi^{2}=a \psi$, (ii) $\chi^{2}=a \chi+b \bar{\chi}$, (iii) $\chi^{2}=a \psi+b \bar{\psi}$, where $\psi \in \operatorname{Irr}(\mathrm{G})$. Here $\bar{\chi}: g \mapsto \overline{\chi(g)}(g \in G)$.

In this note we study the groups $G$ containing a faithful irreducible character $\chi$ taking real values and such that $\left|\operatorname{Irr}\left(\chi^{2}\right)\right|=2$. As Theorem 1.1 shows, this condition is fairly restrictive (moreover, as it is shown in the appendix, there are exactly three groups satisfying this condition).

Our main result is the following

THEOREM 1.1. The following conditions for a group $G$ are equivalent:

(a) There is a faithful irreducible character $\chi$ of $G$ such that $\chi=\bar{\chi}$ and $\left|\operatorname{Irr}\left(\chi^{2}\right)\right|=2$.

(b) The group $G$ is isomorphic to a (finite) nonabelian subgroup $\mathcal{G}$ of $\mathrm{SL}(2, \mathbf{C})$ such that $\mathcal{G}=\left\langle\mathcal{G}-\mathcal{G}_{4}\right\rangle$, where $\mathcal{G}_{4}=\{x \in \mathcal{G} \mid o(x)=4\}$.

Later, in the appendix, we show that $\mathrm{SL}(2,3), \hat{\mathrm{S}}_{4}$ (the representation group of the symmetric group $\mathrm{S}_{4}$ of degree 4 having exactly one involution), and $\operatorname{SL}(2,5)$ are the unique groups satisfying the hypothesis of Theorem 1.1.

In the proof of Theorem 1.1 the following two lemmas play a crucial role.

LEMma 1.2. For a group $G$ and its irreducible character $\chi$ the following conditions are equivalent:

(a) $\chi(1)=2$ and $\operatorname{det}(\chi)=1$.

(b) $\chi^{2}=2 \cdot 1_{G}+\chi^{(2)}$.

Proof. Let $T$ be a representation of $G$ affording our irreducible character $\chi$. Let $g \in G$ and $\{\alpha, \beta\}$ the spectrum of the matrix $T(g)$.

$(\mathrm{a}) \Rightarrow(\mathrm{b})$ : We have $\operatorname{det}(T)=\operatorname{det}(\chi)=1$ and $\alpha \beta=\operatorname{det}(T(g))=1$, by hypothesis. Next, $\chi(g)=\alpha+\beta$ so that

$$
\chi^{2}(g)=(\alpha+\beta)^{2}=\alpha^{2}+\beta^{2}+2 \alpha \beta=\chi\left(g^{2}\right)+2
$$

since the spectrum of the matrix $T\left(g^{2}\right)$ is $\left\{\alpha^{2}, \beta^{2}\right\}$. Therefore, $\chi^{2}=2 \cdot 1_{G}+$ $\chi^{(2)}$, i.e., (b) holds. 
(b) $\Rightarrow(\mathrm{a})$ : Now let $\chi^{2}=2 \cdot 1_{G}+\chi^{(2)}$. Then $\chi(1)^{2}=2+\chi^{(2)}(1)=2+\chi(1)$ so $\chi(1)(\chi(1)-1)=2$, and we get $\chi(1)=2$. If $T, g \in G$ and $\{\alpha, \beta\}$ are as in the first paragraph of the proof, then, by hypothesis,

$$
\alpha^{2}+\beta^{2}+2 \alpha \beta=(\alpha+\beta)^{2}=\chi^{2}(g)=2+\chi\left(g^{2}\right)=2+\alpha^{2}+\beta^{2} .
$$

It follows that $\alpha \beta=1$, i.e., $\operatorname{det}(T(g))=1$, and so $\operatorname{det}(\chi)=1$, hence condition (a) is fulfilled.

REMARK 1.3. The group of Lemma 1.2 is nonabelian since our irreducible character $\chi$ is of degree $2>1$. Besides, for such $G$ we have $\bar{\chi}=\chi$. Indeed, since $\alpha \beta=1$ (see the proof of Lemma 1.2), we get $\beta=\bar{\alpha}$ since $\alpha$ and $\beta$ are roots of unity. Therefore, $\chi(g)=\alpha+\bar{\alpha}$ is real for all $g \in G$, justifying our claim. The same follows from the equality $\chi^{2}=2 \cdot 1_{G}+\chi^{(2)}$. Indeed, by [1, Theorem 4.6.2], we have $\left\langle\chi^{(2)}, 1_{G}\right\rangle \in\{0,1,-1\}$. Therefore,

$$
\langle\chi, \bar{\chi}\rangle=\left\langle\chi^{2}, 1_{G}\right\rangle=\left\langle 2 \cdot 1_{G}+\chi^{(2)}, 1_{G}\right\rangle \geq 2-1>0
$$

so that $\chi=\bar{\chi}$. In that case, $|G|$ is even (Burnside); the same is also holds since $\chi(1)=2$ (Frobenius-Molien).

Lemma 1.4. If $G$ and $\chi$ are as in Lemma 1.2 and, in addition, $\chi$ is faithful, then

(a) $|\mathrm{Z}(G)|=2$.

(b) $G$ has only one involution which is denoted by $u$.

(c) $\mathrm{T}_{\chi}=\{g \in G \mid o(g)=4\}$.

(d) $\chi^{2}=1_{G}+\psi$, where $\psi$ is a character of $G$ of degree 3 , and $\left\langle\psi, 1_{G}\right\rangle=0$. The character $\psi$ is irreducible if and only if $\langle R(\chi)\rangle=G$; here $R(\chi)=$ $G-\mathrm{T}_{\chi}$.

Proof. (a,b) Take $z \in \mathrm{Z}(\mathrm{G})$. Then $|\chi(z)|=\chi(1)=2$ so, in view of $\chi=\bar{\chi}$, by Remark 1.3, we get $\chi(z)=-2$ since $\chi$ is faithful. We have

$$
4=\chi^{2}(z)=2+\chi^{(2)}(z)=2+\chi\left(z^{2}\right)
$$

and we conclude that $\chi\left(z^{2}\right)=2=\chi(1)$. Since $\chi$ is faithful, we get $z^{2}=1$. It follows that $\exp (\mathrm{Z}(\mathrm{G})) \leq 2$. But $\mathrm{Z}(G)$ is cyclic since $\chi$ is faithful, and hence $|\mathrm{Z}(\mathrm{G})| \leq 2$.

Next, there is in $G$ an involution $u$ since $|G|$ is even (see Remark 1.3). By Lemma 1.2(b),

$$
\chi^{2}(u)=2+\chi\left(u^{2}\right)=2+\chi(1)=4 .
$$

It follows that $\chi(u)=-2$, and we conclude that $u \in \mathrm{Z}(\mathrm{G})$ since $\chi$ is faithful. Thus, $|\mathrm{Z}(\mathrm{G})|=2$ and $u$ is the unique involution in $G$. The proof of parts (a) and (b) is complete.

(c) Take $g \in \mathrm{T}_{\chi}$. Then $0=\chi^{2}(g)=2+\chi\left(g^{2}\right)$, i.e., $\chi\left(g^{2}\right)=-2=-\chi(1)$. Therefore, $g^{2} \in \mathrm{Z}(\mathrm{G})^{\#}$ so $g^{2}=u$ (recall that $u$ is the unique involution in $G$, by (b)). It follows that $o(g)=4$. Conversely, if $o(g)=4$, then $g^{2}=u$ and 
$\chi^{2}(g)=2+\chi\left(g^{2}\right)=2+\chi(u)=2-2=0$, i.e., $g \in \mathrm{T}_{\chi}$. The proof of $(\mathrm{c})$ is complete.

(d) Since $\chi$ is real-valued, then

$$
\left\langle\chi^{2}, 1_{G}\right\rangle=\langle\chi, \bar{\chi}\rangle=\langle\chi, \chi\rangle=1 .
$$

Therefore, $\chi^{2}=1_{G}+\psi$, where $\psi$ is a character of $G$ of degree 3 and $\left\langle\psi, 1_{G}\right\rangle=$ 0 .

Suppose that $\psi$ is reducible; then $\psi$ has a linear constituent $\lambda$ since $\psi(1)=$ 3. Since $\langle\psi, \lambda\rangle>0=\left\langle\psi, 1_{G}\right\rangle$, we get $\lambda \neq 1_{G}$, i.e., $G-\operatorname{ker}(\lambda) \neq \emptyset$. Taking into account that $\bar{\chi}=\chi$, we obtain:

$$
\langle\chi, \lambda \chi\rangle=\left\langle\chi^{2}, \lambda\right\rangle=\left\langle 1_{G}+\psi, \lambda\right\rangle=\langle\psi, \lambda\rangle>0
$$

so that $\lambda \chi=\chi$ since the character $\lambda \chi$ is irreducible. Then $\chi\left(\lambda-1_{G}\right)=0$ so that $G-\operatorname{ker}(\lambda) \subseteq \mathrm{T}_{\chi}$, i.e., $R(\chi)=G-\mathrm{T}_{\chi} \subseteq \operatorname{ker}(\lambda)<\mathrm{G}$ since $\lambda \neq 1_{G}$. In particular, $\langle R(\chi)\rangle<G$.

Now suppose that $N=\langle R(\chi)\rangle<G$. We claim that then $g^{2} \in N$ for all $g \in G$. Let $g \in G-N\left(\subseteq \mathrm{T}_{\chi}\right)$. Then $g^{2}=u$ is the unique involution in $G$ (see (c)). Since $|\chi(x)|=2 \neq 0$ for all $x \in \mathrm{Z}(\mathrm{G})$, we have $\mathrm{Z}(G) \subseteq G-\mathrm{T}_{\chi}=\mathrm{R}(\chi)$ so that $\mathrm{Z}(G) \leq N$. It follows that $G / N$ is a non-identity elementary abelian 2-group. Let $N \leq H<G$, where $|G: H|=2$. In that case, there is the unique $\lambda \in \operatorname{Lin}(\mathrm{G})$ such that $\operatorname{ker}(\lambda)=H$. Since $\chi$ vanishes on $G-H=G-\operatorname{ker}(\lambda)$, we get $\chi\left(\lambda-1_{G}\right)=0$ or, what is the same, $\lambda \chi=\chi$. Therefore, since $\bar{\chi}=\chi$, we get

$$
\langle\psi, \lambda\rangle=\left\langle\chi^{2}-1_{G}, \lambda\right\rangle=\left\langle\chi^{2}, \lambda\right\rangle=\langle\chi, \lambda \chi\rangle=\langle\chi, \chi\rangle=1,
$$

i.e., character $\psi$ is reducible. The proof of $(\mathrm{d})$ and thereby the lemma is complete.

Proof of Theorem 1.1. (a) $\Rightarrow(\mathrm{b})$ : By hypothesis, $\left|\operatorname{Irr}\left(\chi^{2}\right)\right|=2$ so that

$$
\chi^{2}=a \phi+b \psi \quad(\phi, \psi \in \operatorname{Irr}(\mathrm{G}) \text { are dictinct, } \mathrm{a}, \mathrm{b} \in \mathbf{N}) .
$$

Since $\bar{\chi}=\chi$, we have, as above,

$$
\left\langle\chi^{2}, 1_{G}\right\rangle=\langle\chi, \bar{\chi}\rangle=\langle\chi, \chi\rangle=1
$$

so that $1_{G} \in\{\phi, \psi\}$. To fix ideas, suppose that $\phi=1_{G}$. Then, by the displayed formula, $a=1$ so that

$$
\chi^{2}=1_{G}+b \psi .
$$

It follows from $(1.1)$ that $\chi(1)^{2}=1+b \psi(1) \geq 2$ so $\chi(1)>1$ and hence $G$ is nonabelian. Then $\mathrm{T}_{\chi} \neq \emptyset$ (Burnside). Let $g \in \mathrm{T}_{\chi}$. Then, by (1.1), $1+b \psi(g)=\chi(g)^{2}=0$, i.e., $b \psi(g)=-1$. Thus, $b$ divides 1 in the ring of all algebraic integers and so in the ring $\mathbf{Z}$. Therefore, $b=1$, so that

$$
\chi^{2}=1_{G}+\psi \text {. }
$$


As we know (see $[\mathrm{BZ}, \S 4.6]), \operatorname{Irr}\left(\chi^{(2)}\right) \subseteq \operatorname{Irr}\left(\chi^{2}\right)$. Therefore, taking into account (1.2), we obtain

$$
\chi^{(2)}=c 1_{G}+d \psi, \quad(c, d \in \mathbf{Z}) .
$$

Since $\chi=\bar{\chi}$, then, by Theorem 4.6.2 in [1], the Frobenius-Schur indicator $c=\left\langle\chi^{(2)}, 1_{G}\right\rangle= \pm 1$. In that case, by (1.3),

$$
\chi(1)=\chi^{(2)}(1)=c+d \psi(1) .
$$

Besides, by (1.2), we obtain $\chi^{2}(1)=1+\psi(1)$. Since $c= \pm 1$, we get

$$
\frac{1}{d}=\frac{\psi(1)}{\chi(1)-c}=\frac{\chi(1)^{2}-1}{\chi(1)-c}=\chi(1)+c .
$$

Since $\chi(1)+c \geq 2+c \geq 1$, it follows that $\frac{1}{d} \in \mathbf{N}$ so $d=1$. Therefore, by the above displayed formula, we also obtain $c=-\chi(1)+1<0$ so that $c=-1$. Thus (see (1.3)), we have

$$
\chi^{(2)}=-1_{G}+\psi .
$$

It follows from (1.2) and (1.4) that

$$
\chi^{2}=1_{G}+\psi=1_{G}+\chi^{(2)}+1_{G}=2 \cdot 1_{G}+\chi^{(2)} .
$$

It follows from (1.5) and Lemma 1.2 that

$$
\chi(1)=2 \text { and } \operatorname{det}(\chi)=1 .
$$

Let, as above, $T$ be a $\mathbf{C}$-representation affording character $\chi$. Since $T$ is faithful, it follows from (1.6) that the mapping $g \mapsto T(g)(g \in G)$ is an isomorphism of $G$ in the special linear group $\operatorname{SL}(2, \mathbf{C})$. Let us denote by $\mathcal{G}$ the image of $G$ in $\operatorname{SL}(2, \mathbf{C})$; then $\mathcal{G} \cong G$ is nonabelian. Let $X_{4}$ be the set of elements of a group $X$ of order $\neq 4$. To prove that $\mathcal{G}=\left\langle\mathcal{G}-\mathcal{G}_{4}\right\rangle$, it suffices to show that $G=\left\langle G-G_{4}\right\rangle$. But this follows immediately from parts (c) and (d) of Lemma 1.4. Indeed, since the character $\psi$ is irreducible, we have $G=\langle R(\chi)\rangle$, by Lemma 1.4(d). On the other hand, by Lemma 1.4(c), $R(\chi)=G-\mathrm{T}_{\chi}=\mathrm{G}-\mathrm{G}_{4}$. Therefore, $G=\left\langle G-G_{4}\right\rangle$, and so condition (b) is fulfilled.

(b) $\Rightarrow$ (a): Suppose that $G \cong \mathcal{G}$, where $\mathcal{G}$ is a finite nonabelian subgroup of $\operatorname{SL}(2, \mathbf{C})$ satisfying $\mathcal{G}=\left\langle\mathcal{G}-\mathcal{G}_{4}\right\rangle$. Then $G=\left\langle G-G_{4}\right\rangle$. Let $T$ be as in the previous paragraph and $g \mapsto T(g)(g \in G)$ an isomorphism of $G$ onto $\mathcal{G}$. Then $T$ is a (faithful) 2-dimensional C-representation of $G$ such that $\operatorname{det}(T(g))=1$ for all $g \in G$. Next, $T$ is irreducible since $G$ is nonabelian. Let $\chi$ be the character of $T$; then $\chi$ is faithful since $T$ is. We have $\chi(1)=$ 2 and $\operatorname{det}(\chi)=1_{G}$, i.e., $G$ satisfied conditions of Lemma 1.2. Then, by Lemma 1.4(d), $\chi^{2}=1_{G}+\psi$, where $\psi$ is a character of $G$. Since $G=\left\langle G-G_{4}\right\rangle$ and, by Lemma 1.4(c), $G-G_{4}=R(\chi)$, we have $G=\langle R(\chi)\rangle$. Therefore, $\psi$ is irreducible (Lemma 1.4(d)), i.e., $\left|\operatorname{Irr}\left(\chi^{2}\right)\right|=2$, and the theorem is proven. 


\section{APPENDIX}

This appendix is written by Y. Berkovich with essential help of I. M. Isaacs (see below).

Our aim is to describe, up to isomorphism, the groups satisfying the hypothesis of Theorem 1.1.

If a group $G$ has a faithful irreducible character $\chi$ of degree 2, then either $G$ has an abelian subgroup of index 2 or $G / \mathrm{Z}(\mathrm{G}) \in\left\{\mathrm{A}_{4}, \mathrm{~S}_{4}, \operatorname{PSL}(2,5)\right\}$, by F. Klein's theorem [1, Theorem 18.1]. It follows from the proof of Theorem 1.1, that if, in addition, the character $\chi$ is such as in part (a) of the theorem, then $\chi^{2}=2 \cdot 1_{G}+\chi^{(2)}($ see $(1.5)$ ). In that case, by Lemma 1.4, we have $|\mathrm{Z}(\mathrm{G})|=2$.

Suppose that $G$ has an abelian subgroup, say $A$, of index 2 . Then $|G|=2 \cdot\left|G^{\prime}\right| \cdot|\mathrm{Z}(\mathrm{G})|$, by [3, Lemma 12.12]. Since $G$ has only one involution (Lemma 1.4(b)), a Sylow 2-subgroup $P$ of $G$ is either cyclic or generalized quaternion. We also have $G=P \cdot B$, where $B$ is a $2^{\prime}$-Hall subgroup of $A$. Since $\mathrm{Z}(G)$ is of order 2 , we get $\left|G: G^{\prime}\right|=2|\mathrm{Z}(\mathrm{G})|=4$ and $\mathrm{N}_{G}(P)=P$. If $P$ is cyclic, then $|P|=4$ and $G$ is not generated by elements of order $\neq 4$, contrary to part (b) of Theorem 1.1. Now assume that $P$ is a generalized quaternion group. Then, if $U / B$ is cyclic of index 2 in $G / B$, then all elements of $G$ of orders $\neq 4$ are contained in $U<G$ so that $G$ is not generated by elements of order $\neq 4$, again a contradiction.

Thus, we must have $G / \mathrm{Z}(\mathrm{G}) \in\left\{\mathrm{A}_{4}, \mathrm{~S}_{4}, \operatorname{PSL}(2,5)\right\}$. Since $\mathrm{Z}(G)$ is of order 2 and generated by unique involution from $G$, it follows that $\mathrm{Z}(G)$ is isomorphic to the Schur multiplier of $G / \mathrm{Z}(\mathrm{G})$ so that $G$ is a representation group of $G / \mathrm{Z}(\mathrm{G})$ (see [BZ, Chapter 6]). It follows that $G \in\left\{\mathrm{SL}(2,3), \hat{\mathrm{S}}_{4}, \mathrm{SL}(2,5)\right\}$, where $\hat{\mathrm{S}}_{4}$ is one of two representation groups of $\mathrm{S}_{4}$ with generalized quaternion Sylow 2-subgroup.

It is easy to check that $\mathrm{SL}(2,5)$ has exactly four faithful irreducible characters of degrees $2,2,4,6$, respectively. It follows that $\mathrm{SL}(2,5)<\mathrm{GL}(2, \mathbf{C})$, so that

$$
\mathrm{SL}(2,5)=\mathrm{SL}(2,5)^{\prime} \leq \mathrm{GL}(2, \mathbf{C})^{\prime}=\mathrm{SL}(2, \mathbf{C}) .
$$

Next, $\operatorname{SL}(2,5)$ is generated by elements of orders $\neq 4$ (otherwise, $\operatorname{SL}(2,5)^{\prime}<$ $\mathrm{S}(2,5))$. It follows that $\mathrm{SL}(2,5)$ satisfies the condition of Theorem 1.1(b). In that case, by Theorem 1.1(a), SL $(2,5)$ has a faithful $\chi \in \operatorname{Irr}(\mathrm{G})$ such that $\chi=\bar{\chi}$ and $\left|\operatorname{Irr}\left(\chi^{2}\right)\right|=2$. Next, SL $(2,3)$ is generated by elements of orders $\neq 4$ and is isomorphic to a subgroup of $\mathrm{SL}(2,5)$ so it is isomorphic to a subgroup of SL $(2, \mathbf{C})$. Then, by Theorem 1.1(a), SL $(2,3)$ has a faithful $\chi \in \operatorname{Irr}(G)$ such that $\chi=\bar{\chi}$ and $\left|\operatorname{Irr}\left(\chi^{2}\right)\right|=2$.

It remains to consider the group $G=\hat{\mathrm{S}}_{4}$ with only one involution. ${ }^{1}$ It is easy to check that $\hat{\mathrm{S}}_{4}$ is generated by elements of orders $\neq 4$, namely, of

\footnotetext{
${ }^{1}$ The result of this paragraph is contained in the letter of Isaacs at August 11, 2009. Isaacs' argument, reworked by Berkovich, is presented below; that argument is based on Isaacs' idea.
} 
orders 3 and 8 . We claim that $\hat{\mathrm{S}}_{4}$ is isomorphic to a subgroup of the special linear group $\mathrm{SL}(2, \mathbf{C})$. Note, that $\hat{\mathrm{S}}_{4}$ has exactly three faithful irreducible characters, and their degrees are $2,2,4$, respectively. Let $\chi \in \operatorname{Irr}(\mathrm{G})$ is faithful of degree 2 and let $T$ be a representation of $G$ affording character $\chi$. Let $P \in \operatorname{Syl}_{2}(\mathrm{G})$; then $P$ is generalized quaternion. We have $P \cap G^{\prime}=Q \cong \mathrm{Q}_{8}$. Since $P$ is generated by elements of order 4 , there is in $P-Q$ an element $x$ of order 4 ; then $x \notin G^{\prime}$. Let $\{\alpha, \beta\}$ be the spectrum of matrix $T(x)$. Since $x^{2}$ is the unique involution in $G$ so that $x^{2} \in \mathrm{Z}(\mathrm{G})$, and the spectrum of $T\left(x^{2}\right)$ is $\left\{\alpha^{2}, \beta^{2}\right\}$, it follows that $T\left(x^{2}\right)=\operatorname{diag}\left(\alpha^{2}, \alpha^{2}\right\}$ is a scalar matrix so that $\alpha^{2}=\beta^{2}$. Since $T$ is faithful, it follows that $\alpha \neq \beta$ so that $\alpha=-\beta$; in that case, $\chi(x)=0$. Since $o(x)=4$, it follows that $\alpha$ is a 4 -th root of unity. Thus, $\alpha \in\{i,-i\}$, where $i=\sqrt{-1}$. In that case, $\operatorname{det}(T(x))=\alpha \beta=i(-i)=1$. Since $T$ is faithful, we get $x \in \mathrm{SL}(2, \mathbf{C})$. By the previous paragraph, $G^{\prime} \cong \mathrm{SL}(2,3)$ and $T\left(G^{\prime}\right)<\mathrm{SL}(2, \mathbf{C})$. Thus, $T(G)=\left\langle T(x), T\left(G^{\prime}\right)\right\rangle<\mathrm{SL}(2, \mathbf{C})$. We see that $G$ satisfies the condition of Theorem 1.1(b). Therefore, since parts (a) and (b) of the theorem are equivalent, it follows that $\bar{\chi}=\chi$ and $\left|\operatorname{Irr}\left(\chi^{2}\right)\right|=2$.

REMARK 2.1. Let $H$ be the representation group of $\mathrm{S}_{4}$ with semidihedral Sylow 2-subgroup (in that case, $H \cong \mathrm{GL}(2,3)$ ). It follows from the above that $\mathrm{SL}(2, \mathbf{C})$ has no subgroup isomorphic to $H$ (this can be checked directly).

Thus, now Zhmud's Theorem 1.1 can be stated in the following form:

A group $G$ has a faithful irreducible character $\chi$ such that $\bar{\chi}=\chi$ and $\left|\operatorname{Irr}\left(\chi^{2}\right)\right|=2$ if and only if $G$ has only one involution and $G \in$ $\left\{\mathrm{SL}(2,3), \hat{\mathrm{S}}_{4}, \mathrm{SL}(2,5)\right\}$.

Let us present an irreducible character $\chi$ of $G=\hat{\mathrm{S}_{4}}$ such as in Theorem 1.1. It follows from the character table of $G$ that it has two irreducible characters $\chi$ and $\psi$, taking on the corresponding $G$-classes the following values:

Then

$$
\begin{gathered}
\chi: 2,-2,-1,0,0,1, \sqrt{2}, \sqrt{2} \\
\psi: 3,3,0,-1,-1,0,1,1 .
\end{gathered}
$$

$$
\begin{gathered}
\chi^{2}: \quad 4,4,1,0,0,1,2,2, \\
1_{G}+\psi: 4,4,1,0,0,1,2,2 .
\end{gathered}
$$

We see that $\chi^{2}=1+\psi$.

Old PROBlem. Classify the groups $G$ containing a faithful irreducible character $\chi$ such that $\left|\operatorname{Irr}\left(\chi^{2}\right)\right|=2$ (Theorem 1.1 solves this problem for real-valued $\chi$ ).

ACKNOWLEDGEMENTS.

Y. Berkovich is indebted to Martin Isaacs for help in completing this note and useful discussion of questions concerning to character tables of $\hat{\mathrm{S}}_{4}$ and other related groups, and the referee for a number of useful remarks. 


\section{REFERENCES}

[1] Y. G. Berkovich and E. M. Zhmud, Characters of Finite Groups. Parts 1 and 2, Translations of Mathematical Monographs 172 and 181, AMS, Providence, RI, 1998.

[2] H. Blau and D. Chillag, On powers of characters and powers of conjugacy classes of a finite group, Proc. Amer. Math. Soc. 98 (1986), 7-10.

[3] I. M. Isaacs, Character theory of finite groups, Academic Press, New York, 1976.

[4] I. M. Isaacs and I. Zisser, Squares of characters with few irreducible constituents in finite groups, Arch. Math. (Basel) 63 (1994), 197-207.

Received: 12.8 .2009 .

Revised: 4.9.2009. 